\title{
Magnetic Resonance Guided Radiofrequency Ablation: Creation and Visualization of Cardiac Lesions
}

\author{
Albert C. Lardo, Ph.D., Henry Halperin, M.D., Christopher Yeung, BA.Sc., \\ Pitayadet Jumrussirikul, M.D., Ergin Atalar, Ph.D., Elliot McVeigh, Ph.D. \\ Johns Hopkins School of Medicine \\ Departments of Biomedical Engineering, Cardiology, and Radiology \\ Baltimore, M.D. 21205
}

\begin{abstract}
Current management of atrial fibrillation (AF) refractory to pharmacological therapy involves the creation of lines of conduction block in the atrial tissue either by direct surgical incision or fluoroscopy guided radiofrequency (RF) ablation. While these techniques have been shown to be effective in terminating atrial fibrillation, open heart surgery carries considerable inherent risk and fluoroscopy guided approaches are technically difficult and result in extremely long radiation exposure times. Magnetic resonance (MR) guided ablation therapy may represent a safer and more practical alternative for creating lines of conduction block in patients with AF. We have developed a novel MR compatible system that permits simultaneous high resolution MR imaging, cardiac electrical mapping, and RF energy delivery. This system has, for the first time, made it possible to perform a comprehensive MR guided electrophysiology study including guidance, delivery, and monitoring of cardiac ablation therapy.
\end{abstract}

\section{Introduction}

Atrial fibrillation is a common cardiac arrhythmia that affects over 2 million people in the United States and is characterized by rapid, random, and hemodynamically inefficient atrial contractions that result in 75,000 stokes per year [1]. In patients unresponsive to drug therapy, AF management is achieved by the surgical maze procedure [2] where strategically placed long linear atrial incisions are created to compartmentalize the electrical activity of the atrial chamber and terminate abnormal excitation pathways. While the efficacy of this procedure has been demonstrated [3], the procedure is technically difficult and involves significant operative risk. A less invasive alternative designed to mimic the surgical maze procedure has been attempted using fluoroscopy guided radiofrequency ablation therapy, where electrical energy is deposited into the tissue and localized RF induced hyperthermia results in irreversible cellular destruction and elimination of abnormal electrical propagation pathways. While early experience with this technique appears 
promising, the success of fluoroscopy guided cardiac ablation is limited by the inability to create and visualize linear and contiguous (incision-like) lesions, which in turn leads to extremely long procedure times, ineffective treatments, and excessive $\mathrm{x}$-ray exposure for both the patient and physician. In light of these limitations, the development of an alternative interventional approach is warranted.

MR guided thermal therapy delivery may be a logical alternative to fluoroscopic techniques as it offers several theoretical and practical advantages including: 1) elimination of patient and physician exposure to ionizing radiation, 2) rapid three-dimensional visualization of cardiac chambers and thermal lesions with high spatial resolution, 3) real time catheter placement with a potential reduction in the number of required surgical lesions and a corresponding reduction in procedure time, 4) simultaneous high resolution imaging, electrical mapping and RF delivery, 5) potential for real time spatial temperature monitoring using temperature sensitive imaging protocols and T1/T2 signal changes in heated tissue, and 6) high resolution atrial imaging to evaluate function and flow dynamics during $\mathrm{AF}$ which may provide an assessment of future risk of embolization. We have developed a novel MR compatible hardware system for use with a loopless intracardiac MR imaging catheter previously described by our group [4], to perform a comprehensive MR guided interventional electrophysiology study. While previous studies have described the use of MR guided thermal therapies for the control of abdominal, prostate, and liver tumors, to date no studies have demonstrated the ability to create and visualize cardiac RF ablation lesions using interventional MRI. Accordingly, the purpose of this study was to develop and characterize a novel MR ablation/antenna system capable of high-resolution intracardiac imaging for guidance, delivery, and monitoring of cardiac radiofrequency thermal therapy.

\section{Methods and Materials}

Under fluoroscopic guidance, a 3F imaging antenna and a $5 F$ MR compatible ablation catheter were placed in the right atrium and right ventricle of healthy dogs via the right femoral and jugular veins respectively. RF ablation was performed on the posterior wall of the right atrium and the right ventricular apex using a customized MR compatible $4 \mathrm{~mm}$ electrode tip catheter and clinical ablation unit (Medtronic Atakar ${ }^{(8)}$, Minneapolis, MN) over a range of clinically used powers $(10-50 \mathrm{~W})$ and a constant duration of $60 \mathrm{sec}$. Under MR guidance, several RF burns were delivered to both the posterior-lateral wall of the right atrium and right ventricular apex. Catheter and antenna guidance imaging was performed using the cardiac phased array coil in a 1.5 T closed system (Signa 5.0, General Electric, Milwaukee, WI) with a gradient echo imaging protocol $(\mathrm{TR} / \mathrm{TE}=1.0 / 1.2 \mathrm{~ms}$, field of view $=24$ 
$\mathrm{cm}$, slice thickness $=8 \mathrm{~mm}, 256 \times 192$ matrix, tip angle $=11$ degrees, readout bandwidth $=64 \mathrm{kHz}$ ). Following each 60 -second burn session, lesion imaging was performed every three minutes with the cardiac phased array coil and antenna using both $\mathrm{T} 1$ gradient echo and $\mathrm{T} 2$ weighted fast spin echo sequences $(\mathrm{TE}=50.8 \mathrm{~ms}, \mathrm{ETL}=8$, field of view $=14 \mathrm{~cm}$, slice thickness $=6$ $\mathrm{mm}, 256 \times 128$ matrix, readout bandwidth $=62.5 \mathrm{kHz}$ ) to characterize temporal and spatial MR signal changes of the cardiac tissue following RF injury. To compare the MR lesion appearance and size with the anatomical lesion, the heart was excised immediately following sacrifice and inspected. Lesions in the right atrium and ventricle were photographed and matched with the corresponding lesions from $\mathrm{T} 2$ weighted fast spin echo images.

\section{MR Imaging Antenna}

The intravascular imaging catheter used in these studies consists of a dipole antenna constructed from a $1.2 \mathrm{~mm}$ diameter segment of co-axial semiflexible cable and MR compatible matching, tuning and decoupling circuitry [4] (Figure 1). The outer conductor of the cable, which is used to carry the MR signal to the antenna circuitry, serves as one pole, while the inner conductor serves as the second pole that receives the MR signal generated following body coil excitation of the slice. Because of the extremely high sensitivity and SNR in the immediate vicinity of the antenna, near microscopic resolution is a possible which is ideally suited for visualization of small thermal RF lesions. This major advantage is in addition to the excellent tissue characterization inherent to MRI.

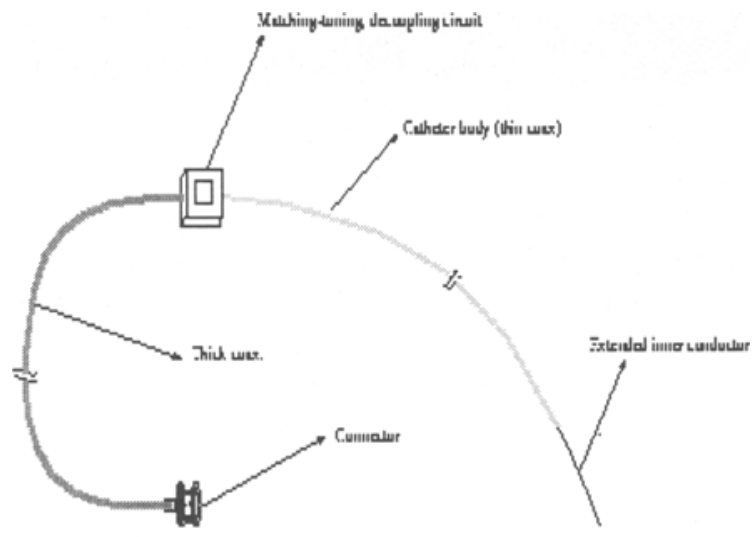

Figure 1 Schematic of the dipole imaging antenna. ${ }^{4}$ 


\section{MR Compatible Filters}

One of the problems associated with simultaneous radiofrequency energy delivery and MR imaging is electromagnetic interference that results in interference with the MR image. While the frequency of the radiofrequency generation unit $(550 \mathrm{kHz})$ is well below the $64 \mathrm{MHz}$ proton precession frequency at $1.5 \mathrm{~T}$, higher harmonics of the radiofrequency signal can produce significant image degradation. To overcome this limitation, novel MR compatible filters were designed and constructed to suppress these higher harmonic signals and permit simultaneous RF ablation and electrophysiology monitoring during MR imaging. These multi-stage, low-pass filters consist of a strategic arrangement of electrical components that achieve a cut off frequency of approximately $10 \mathrm{MHz}$. Figure 2 shows an example of the dramatic effect of the RF ablation signal on image quality as the left panel represents the MR image during RF delivery without filtering while the image on the right shows the same slice during RF delivery with filtering. Note there is no evidence of noise or artifact and the tip of the ablation catheter is clearly visible.
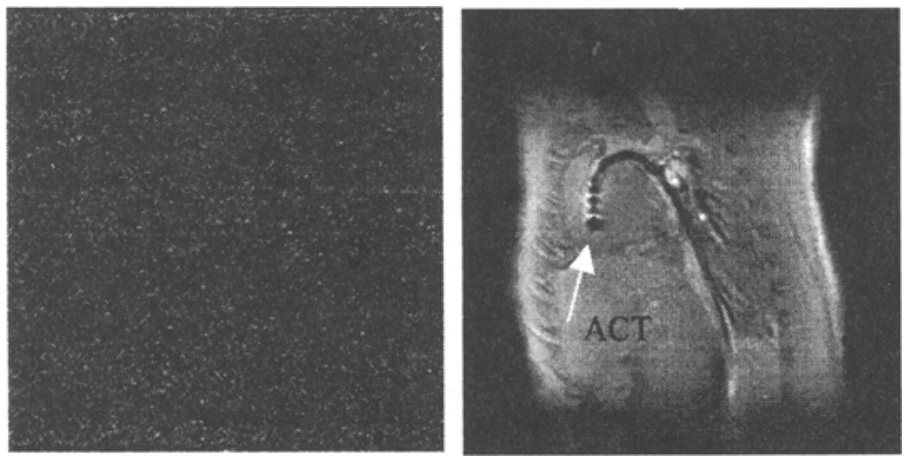

Figure 2 Sagital phased array coil images during RF ablation a) without and b) with filtering. Note the clear visualization of the ablation catheter tip (ACT) in the right ventricle.

\section{Results}

A representative gradient echo antenna catheter image of the right atrium is shown in Figure 3 during a $30 \mathrm{~W} 60 \mathrm{sec}$ radiofrequency delivery (FOV $=14$ $\mathrm{cm})$. The MR compatible ablation catheter $(\mathrm{AC})$ is shown on the lateral wall of the right atrium (arrow) directly adjacent to the imaging antenna. Note 
that the superior vena cava and entire atria is illuminated exclusively by the antenna catheter. Figure 4 shows a post-mortem coronal fast spin echo image

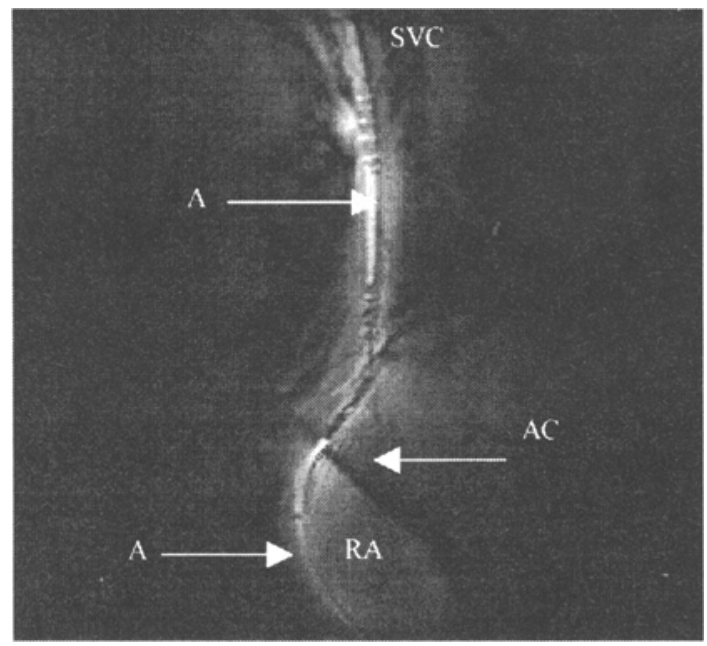

Figure 3 Antenna image showing the placement of the antenna (A) and ablation catheter $(\mathrm{AC})$ in a canine right atrium (field of view = $14 \mathrm{~cm})$.

through the atria and ventricle of a dog acquired using the cardiac phased array coil, with the intracardiac antenna coil image shown in the enlarged window "C". The ablated areas of the myocardium can be visualized (arrows) in the intraventricular septum (IVS) and the enlarged area of the RA. Blood in the RA and RV is shown in white. Figure 5 shows a series of fast spin echo images of a right ventricular apex lesion before and after RF three $\mathrm{RF}$ burns. The first image of the series represents the baseline image before RF delivery with the arrow indicating the position of ablation catheter. The second image is $5 \mathrm{~min}$ following two $10 \mathrm{~W} 30 \mathrm{sec}$ ventricular burns. Note the hyperintense region at the tip of the ablation catheter (arrow). The third image is the same slice imaged 10 min following three $25 \mathrm{~W} 60 \mathrm{sec}$ RF burns. The hyperintense region around the ablation catheter tip in the right ventricular apex is more pronounced in both intensity and size. 


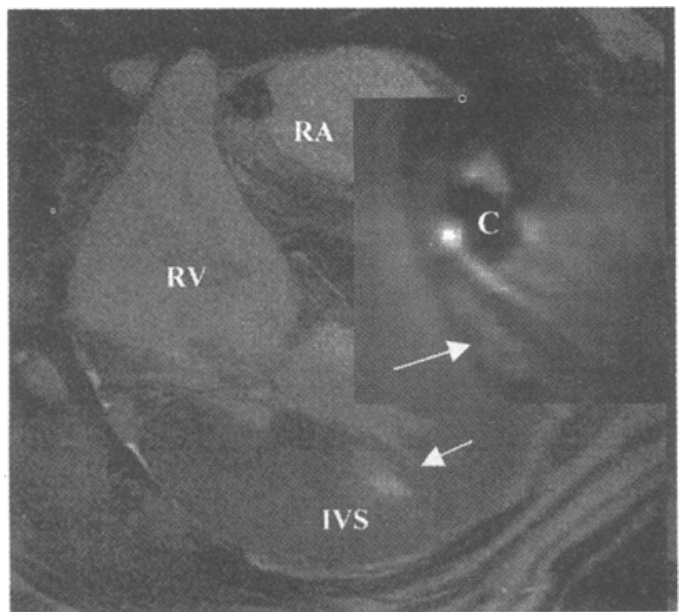

Figure 4 Coronal fast spin echo image of atria and ventricle following RF ablation with the torso and antenna coil "C".
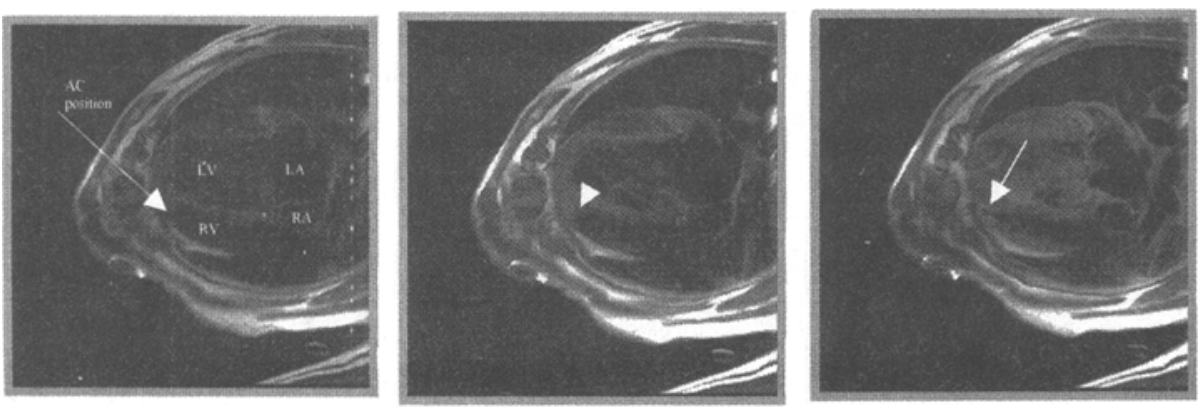

Figure 5 T2 weighted fast spin echo images showing right ventricular apex slices a) at baseline and following $60 \mathrm{sec}$ RF energy delivery at b) $10 \mathrm{~W}$ and c) $30 \mathrm{~W}$. Note the increase in lesion size between b) and c). 


\section{Discussion}

Over the past several years, MRI guided thermal therapy has slowly evolved from a theoretical research tool to a clinically applied treatment that has shown significant promise as an alternative to traditional invasive surgical procedures and radiology imaging techniques. The ongoing maturity of this exciting interventional tool has largely been driven by applications related to the local control of regional metastic cancer in most major organs. Despite the recent advent of cardiac ablation techniques, which have revolutionized the management of patients with supraventricular arrhythmias refractory to pharmacological therapy, MRI guidance of thermal therapy in the heart has received virtually no attention. This is most likely attributed to the many challenges inherent to interventional MR cardiac imaging, including the geometric complexity of the heart, significant organ motion, presence of high blood flow, and the need for rapid imaging and display. In this study, we describe a system designed to permit MR guided cardiac thermal therapy and thus potentially serve as an alternative to surgical and fluoroscopy guided ablation techniques for atrial fibrillation and other cardiac arrhythmias. These encouraging, but preliminary results indeed indicate that MRI may be used to guide cardiac thermal therapy, although further work is required to determine the direct applicability and utility of this exciting technique.

\section{Conclusions}

These preliminary studies have demonstrated that it is possible to perform RF tissue ablation during MR imaging without compromising SNR. The tip of the catheter is clearly defined in gradient echo images and ablation lesions could be visualized in the right atrium and ventricle using T2 weighted FSE imaging with the both the intracardiac antenna and cardiac phased array coil. MR guided ablation may be a logical alternative to fluoroscopy guided techniques that will eliminate radiation exposure and may potentially reduce procedure times by facilitating the creation of linear, contiguous and transmural atrial lesions required for AF termination. Additionally, accurate visualization of the areas of ablation may allow for a reduction in the total number of lesions needed, which may also reduce the reoccurrence of $\mathrm{AF}$. 


\section{References}

1. Zipes D. Atrial Fibrillation: A tachycardia-induced atrial cardiomyopathy. Circulation 1997;95:562-564.

2. Cox J, Jaquiss R, Schuessler R, Boineau J. Modification of the maze procedure for atrial flutter and fibrillation, II: surgical technique of the maze II procedure. J Thorac Cardiovasc Surg 1995;1 10:485-495.

3. Haissaguerre M. Right and left atrial radiofrequency ablation catheter therapy of paroxysmal atrial fibrillation. J Cardiovasc Electrophysiol 1996;7:1132-1144.

4. Ocali $\mathrm{O}$, Atalar E. Intravascular magnetic resonance imaging using a loopless catheter antenna. Magn. Reson. Med. 37, 112-118 